Beobachtungen von kleinen Planeten auf der Hamburger Sternwarte im Winterhalbjahr 1868 -1869. Mitgetheilt ron dem Director, Herrn G. Riumker.

Diese Beobachtungen sind Differenzenmessungen am Fadenmikrometer des Aequatoreals. Jede Vergleichung in AR beruht auf durchschnittlich 4 Antritten beider Objecte. In der Columne Beobachtung - Rechnung ist das Resultat der
Vergleichung der Beobachtungen mit den betreffenden Ephemeriden des Berliner Jahrbuchs, für Undina mit derjenigen in den Astronomischen Nachrichten M\& 1717 veröffentlichten, niedergelegt.

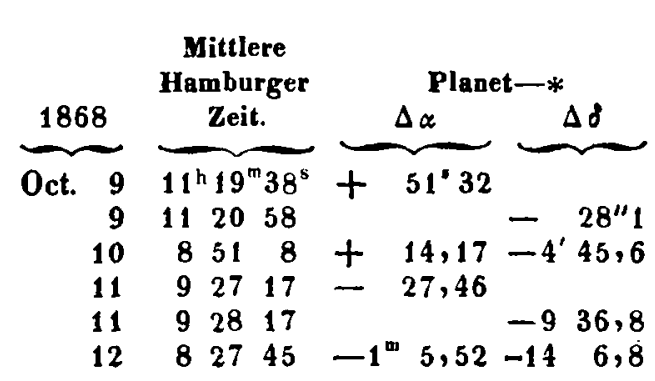

Oct. $912216-50,27-30,8$ $\begin{array}{llllllllll}10 & 8 & 33 & 40 & -1 & 35,16 & -3 & 21,5\end{array}$

$\begin{array}{llllllllll}10 & 10 & 12 & 21 & -1 & 38,92 & -3 & 34,5\end{array}$

$\begin{array}{llllllll}11 & 10 & 6 & 6 & -2 & 33,18 & -6 & 27,3\end{array}$

$22101422-10,36-22,5$

Oct. $9 \quad 13 \quad 15 \quad 12+2 \quad 9,30$

$\begin{array}{lllll}9 & 12 & 13 & 31\end{array}$

$10 \quad 94233+119,16$

$109+8 \quad 1 \quad-352,4$

$11105229+16,99-63,5$

$12102729+6,53+157,6$

$\begin{array}{lllllll}22 & 10 & 37 & 7 & -1 & 16,45+3 & 10,9\end{array}$

Nov. $5105715+15,89-759,9$

Oct. $913548+125,36+127,2$ $101088+38,10-1,2$

$11 \quad 111757-21,50-157,5$

$\begin{array}{lllllllll}12 & 11 & 16 & 56 & -1 & 19,02 & -3 & 54,6\end{array}$

$2211046+51,52+430,2$

Nov. $5103428+318,24+151,6$

Oct. $1292832-328,01+511,4$ $229506+20,69-547,9$

Oct. $22 \quad 9 \quad 1639+243,39+821,9$ Nov. $5 \quad 954 \quad 15-3 \quad 30,51-1 \quad 9,2$

Nov. $5113811+329,84+534,2$ 1869

März $6 \quad 131546-30,94$

$\begin{array}{lllllll}6 & 13 & 19 & 47 & -2 & 8,84\end{array}$

$\begin{array}{lllrllll}10 & 11 & 22 & 9 & -1 & 17,78 & & \end{array}$

$\begin{array}{llll}10 & 11 & 38 & 12\end{array}$
(49) $\mathrm{P}$ a $\mathrm{l}$ e s.

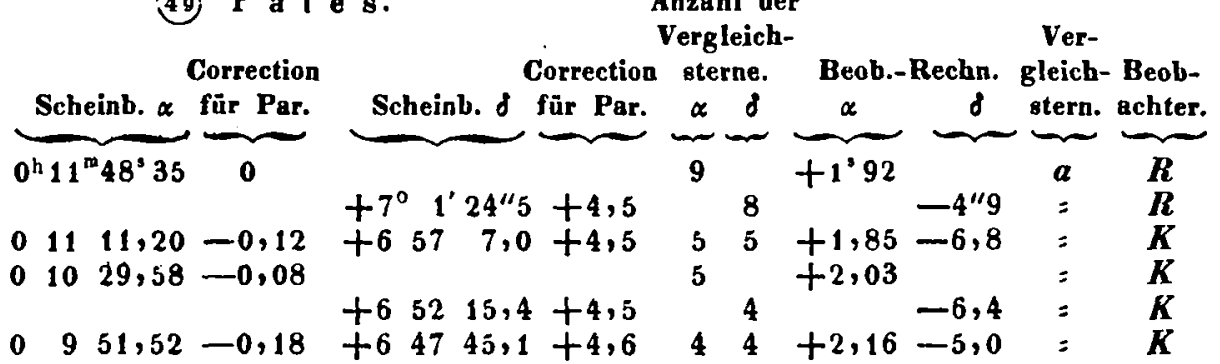

(38) F i d e s.

$1247,33+0,02+10 \quad 639,2+4,6 \quad 9 \quad 9-0,14-0,8 \quad b \quad R$

$12322,45-0,17+10349,5+4,8$

$12318,69-0,12+10335,5+4,7$

$12224,44-0,12+10$ 0 $12,3+4,6$

$11224,04-0,06+92024,5+4,7$

(42) $I \mathrm{~s}$ i s.

$12528,82+0,09$

$12438,69-0,18$

$-72535,5+6,7$

$12336,53-0,09-72746,6+6,8$

$12238,07-0,12-72940,0+6,8$

$1139,29-0,03-73533,5+6,6$

$1256,06+0,07-7$ o $39,2+6,0$

(29) A m p it rite.

t $4738,59+0,09+162634,5+3,7$

$14651,34-0,14+16256,2+3,9$

$14551,75-0,07+162310,0+3,7$

$14454,24-0,07+162112,9+3,7$

$1350,78-0,03+155526,3+3,8$

$12211,49+0,01+15950,3+3,7$

(10) $\mathrm{H}$ y $\mathrm{g}$ e a .

$1718,93-0,08+12576,8+2,4 \quad 3 \quad 3+0,48+0,2 \quad l \quad K$ $100,91-0,04+12920,6+2,4$

(12) Vi c t o r i a.

$1477,16-0,18+175951,0+4,5 \quad 4 \quad 4-6,66-32,0$ n $K$

$13456,68-0,06+153515,6+4,2 \quad 3 \quad 3-5,71-33,8$ o $K$

92. Und i u a.

$3155,78-0,03+52528,9+3,1 \quad 1 \quad 1-39,12-2^{\prime} 0^{\prime \prime} 1 \quad p \quad K$

(62) $\mathrm{E} u \mathrm{r}$ o $\mathrm{p}$ a.

$105710,62+0,08$

$105419,12 \quad 0$

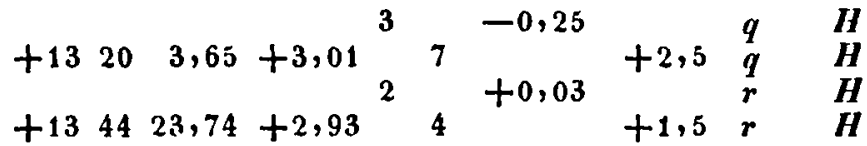

Beobachter: Director Rïmker $=\boldsymbol{R}$, Dr. Kampf $=\boldsymbol{K}$, Dr. Helmert $=\boldsymbol{H}$. 


\section{Positionender Vergleichsterne.}

\begin{tabular}{|c|c|c|c|c|c|c|c|c|}
\hline$a$ & Weiss & 169 & Mittl. & Ort & 1868,0 & $0^{\mathrm{h}} 10^{\mathrm{m}} 54^{\mathrm{s}} 05$ & $+7^{\circ}$ & $1^{\prime} 33^{\prime \prime \prime} 5$ \\
\hline b & $=$ & 409 (Gew. 2); Lal. 2775 (Gew. 1) & $=$ & $=$ & $=$ & 12454,50 & +10 & 654,7 \\
\hline 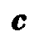 & $=$ & 172 (1) ; Berl. Mer.-Beob. Astr. Nachr. Bd. 64, p. 277 (3) & $=$ & $=$ & $=$ & 11231,28 & +92 & 2028,8 \\
\hline d & $=$ & 383 & $=$ & $=$ & $=$ & $1,23 \quad 16,65$ & -72 & 2158,6 \\
\hline $\boldsymbol{e}$ & $=$ & 363 (2); Lal. 2719 (1); Rutmk. N. F. 688 (4); 2 Bonner B. (B) & ) & $=$ & $=$ & 12228,60 & -73 & 3153,1 \\
\hline$f$ & $=$ & 212 & $=$ & $=$ & $=$ & 11422,62 & -73 & $39 \quad 2,6$ \\
\hline 9 & $=$ & 1082 & $=$ & $=$ & $=$ & 1147,26 & -6 & 5253,1 \\
\hline$h$ & $=$ & 1053 (1); Rumker N. F. 952 (2) & $=$ & $=$ & $=$ & $146 \quad 10,02$ & $+16 ?$ & 2453,8 \\
\hline$i$ & $=$ & 768 (2); Lal. 3088 (1) & $=$ & $=$ & $=$ & $134 \quad 5,99$ & +15 & 5040,8 \\
\hline
\end{tabular}

Nach Zone 394 ist die Decl. bei W. $1^{\prime}$ zu gross. Die reducirten Oerter weichen in AR um $1^{\text {s }}$, in Decl. um $4^{\prime \prime}$ von einander ab.

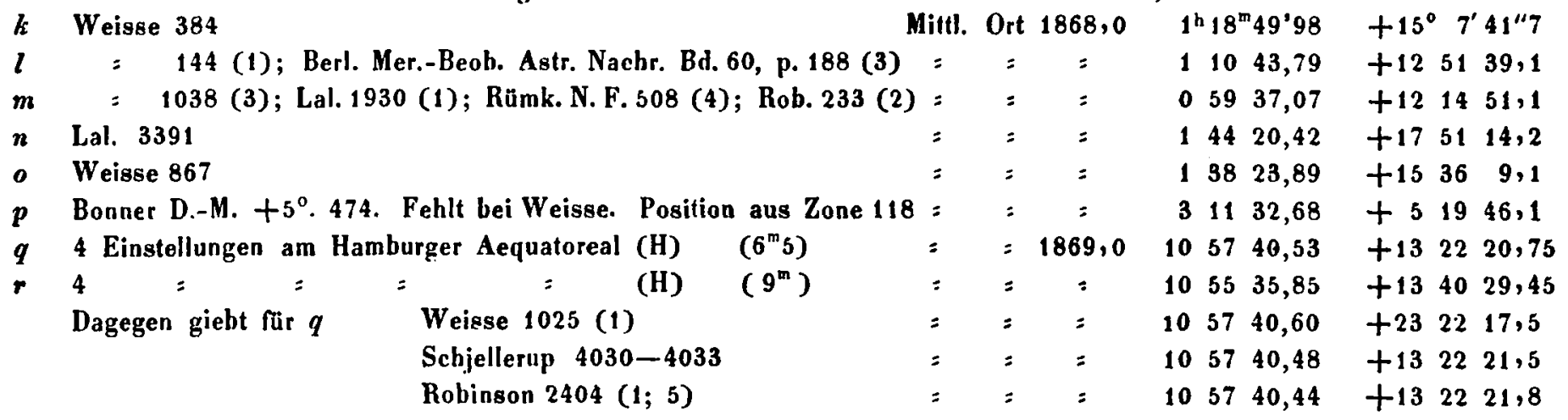

doch wurde es vorgezogen, die Aequatoreal-Beobacbtungen einzuführen.

Der Unterschied der beiden Victoria-Beobachtungen kann wohl nur durch einen Febler in der Ephemeride erklärt werden, doch könnte auch der Vergleichstern $n$ in AR feblerhaft sein, da hiervon nur ei n Fadendurchgang bekannt ist.

Am Aequatoreal wurde die Venus - Bedeckung durch den Mond beobachtet am 1868 October 12 . Eintritt: $3^{\mathrm{h}} 29^{\mathrm{m}} 22^{\prime} 7$ mittl. Hamb. Zt.

und der Durchgang des Mercur über die Sonne, wenn auch wegen Wolken nur theilweise,

1868 November 4. Erste Berührung des Austritts. $21^{\mathrm{h}} 39^{\mathrm{m}} 57^{\mathrm{s}}$ mittl. Hamb. Zt. (K)

Mitte des Austritts (unsicher) $214124=\leq=$

bei 300 maliger Vergrösserung.

\section{Beobachtung des Winnecke'schen Cometen, von Herrn Dr. E. Weiss.}

1869 April 29, $9^{\mathrm{h}} 55^{\mathrm{m}} 20^{\circ} \mathrm{m}$. Zt.Wien. Sch. $\alpha=10^{\mathrm{h}} 0^{\mathrm{m}} 24^{\circ} 2$, l. f. p. $=8,518$; sch. $\delta=+36^{\circ} 34^{\prime} 39^{\prime \prime}$, I. f. p. $=9,446$.

Die Beobachtung ist wegen der Schwäche des Cometen, und wegen seines ungemein verwaschenen Aussehens, beim Mangel eines jeden Kernes sebr unsicher.

Wien, 1869 April 29.

Ed. Weiss.

\section{Fortsetzung der Ephemeride für den Winnecke'schen Cometen.}

Anbaltend ungünstige Witterung hat in Pulkowa bis beute jede Beobachtung des Winnecke'schen Cometen unmöglich gemacht. Herr Dr. Winnecke ist aber so gütig gewesen, mir drei von ihm erhaltene genäherte Positionen von April 9, 10, 11, mitzutheilen, welche ich dazu benutzt habe, die wahre Perihelzeit wenigstens mit einer solchen Annäherung herzuleiten, dass die darauf basirte Ephemeride den Cometenort mit einer Genauigkeit angiebt, die das mübelose Auffinden des Objectes ermöglicbt.

Da ich bis jetzt noch nicht dazu gebommen bin, die Herleitung meiner Elemente ausfthihrlich zu hegründen, so führe ich hier wenigstens einstweilen die hauptsächlichsten Zablen aus meines Rechoungen auf, die vielleicht mancher Beobachter des Cometen zu haben wünscht. 